\title{
Discrete time minimax tracking control with disturbance estimation
}

\author{
Péter Bauer, Balázs Kulcsár and József Bokor
}

\begin{abstract}
The paper proposes an alternative way to solve robust reference tracking problem. Instead of rejecting the effect of the disturbance directly, an intermediate step is built into the state estimation problem. The advantage of the methodology is to elaborate a modified optimal state estimation problem taking the unbiased estimate of the disturbance into account. Henceforward, the solution of the discrete time, optimal LQ minimax tracking problem is modified and subjected to attenuate the disturbance residual. The paper addresses the nominal case for constant reference and disturbance signal showing the asymptotical stability and the tracking performance of the discrete time, optimal min-max control. Suboptimal solution is given for time varying reference and disturbance signal. A linearized hovering quadrotor example demonstrates the importance of the suggested technique.
\end{abstract}

Index Terms-LQ optimal minimax tracking, state and disturbance estimation

\section{INTRODUCTION}

Robust tracking control solutions with output feedback make use of the systems' estimated states [7]. Here, the objective is to attenuate the disturbance level on the performance output by introducing and minimizing the induced $\mathcal{L}_{2}$ norm. Accordingly, the disturbance rejection property plays an important role in minimax respectively $\mathcal{H}_{\infty}$ control techniques. If the disturbance lies in the low frequency range, difficulties arise in providing acceptable tracking performance, since there is a trade-off in tuning the sensitivity respectively complementary sensitivity functions. The literature proposes solutions in the preview control field with disturbance preview (see ex. [2], [3], [4], [5], [6]), sometimes even with reference anticipation ([5],[6]). Consequently, there is a high demand on the estimation of the disturbance allowing accurate reference tracking.

Similarly to [8], our goal is to eliminate as much of the effects of the deterministic disturbance as we can, based on coupled state and disturbance estimation (see [10], [9]). The stabilizing minimax control has to guarantee zero steady state tracking error (at least for constant reference and disturbance signals) and has to be be optimal over an infinite horizon for constant signals. The motivation of the work is to develop optimal and tracking control solutions when deterministic

The authors are partially with the Systems and Control Laboratory, Computer and Automation Research Institute, Hungarian Academy of Sciences, Budapest, H-1111, Hungary \{bauer.peter, bokor\}esztaki.hu, and partially with the Delft Center for System and Control, Faculty of Mechanical, Maritime and Materials Engineering, Technical University of Delft Mekelweg 2,Delft, Nederland b.a.kulcsardtudelft.nl

The authors gratefully acknowledge the support of the Hungarian National Science foundation (OTKA CNK78168), Control Engineering Research Group of H.A.S. at Budapest University of Technology and Economics and the TRUCKDAS project (TECH_08-A2/2-2008-0088, OM-00239/2008) (and mostly constant or slowly varying) disturbance perturb the plant. The disturbance effect might be considered as a quasi stationary load, therefore an adequate solution is certainly to increase the number of the state in the state space by co-state variables. The co-state variables assigns dynamics to the exogenous disturbance signal under the form of a random walk model. However, our approach is different from the above mentioned state augmentation problem. The core of the idea is to compute the estimated and unbiased disturbance value of the previous step and actualize it either by taking it constant or filter it through an a-priori known dynamics. Even if the disturbance reconstruction is unbiased, due to the transient behavior, the disturbance residual could influence the performance a lot. Therefore, the elaborated minimax control solution has to reject the value of the disturbance in the transient phase. These goals can be achieved through a multi step design procedure explained further into the details. The properties of the resulted control technique are examined for constant and partially time-varying reference and disturbance signals.

The paper is organized as follows. In Section II the problem is formulated and the steps of the proposed multi step solution are listed. In section III, the solution steps are detailed. In Section IV, the properties for constant references and disturbances are stated and proven. In the Section V,the method is compared with an $\mathcal{H}_{\infty}$ design through a simple example. Finally, Section VI concludes the paper.

\section{Problem Formulation AND the StePS OF THE PROPOSED SOLUTION}

The considered system class is DT, LTI systems with deterministic disturbances:

$$
\begin{aligned}
x_{k+1} & =A x_{k}+B \tilde{u}_{k}+G d_{k} \\
y_{k}^{r} & =C_{r} x_{k} \\
y_{k} & =C x_{k} \\
d_{k+1} & =A_{y_{k}}^{d} d_{k} \quad\left\|A_{y_{k}}^{d}\right\|_{2}<\infty
\end{aligned}
$$

Where $x_{k} \in \mathbb{R}^{n}, \quad \tilde{u}_{k} \in \mathbb{R}^{m}, \quad d_{k} \in \mathbb{R}^{d} \quad y_{k}^{r} \in \mathbb{R}^{r} \quad y_{k} \in$ $\mathbb{R}^{p}$ are the system state, input, disturbance, tracking output and measured output respectively and $A, B, G, C_{r}, C$ have appropriate dimensions. The last equation shows that the disturbance is assumed to be characterized by an autonomous dynamical system which depends on the measured system outputs. This assumption can be applied in considering the wind disturbances on a moving aerial vehicle for example.

Assumption 1: Assume that $n \geq m, n>d, r \leq m$, $p>d, \operatorname{rank}(C G)=\operatorname{rank}(G)$, the pair $(A, B)$ is stabilizable and the pair $(C, A)$ is observable. Assume also that $\operatorname{rank}\left(C_{r} B\right)=r$. 
The goal is to track a prescribed constant or time-varying reference signal with maximum disturbance attenuation (minimum tracking error). The developed multi-step solution is similar to the method applied in [8]. The steps of the solution are as follows:

1) Design a stabilizing state feedback control input for system (1). This makes step 2 and 4 feasible.

2) Design the optimal state and disturbance estimator for the stabilized system (the method described in [10] and [9] is modified to be used for noiseless LTI systems)

3) Construct the system input which cancels the disturbance effects in a LS optimal way.

4) Design another control input, which guarantees zero steady state tracking error in case of constant reference and disturbance signals.

5) Center the original system (constructed in step 1) dynamics (this is suggested in [1]) with the steady state equilibrium point achieved in the previous step, and design an LQ optimal minimax tracker for this centered dynamics (at first, for finite and then for infinite horizon).

6) Construct the final required input signal $\tilde{u}_{k}$ summing up all the inputs designed in the previous steps.

In the next Section the above steps will be followed to construct the final optimal (for constant references and disturbances) and sub-optimal (for time-varying references and disturbances) controllers.

\section{THE STEPS OF THE DESIGN PROCEDURE}

Step1: Design of a stabilizing state feedback controller for $(A, B)$

This can be solved either with pole placement or with LQ optimal regulator design. The resulting system equations can be written as follows (considering additional input to guarantee tracking):

$$
\begin{aligned}
\tilde{u}_{k} & =-K_{x 1} x_{k}+u_{k} \\
x_{k+1} & =\underbrace{\left(A-B K_{x 1}\right)}_{\phi} x_{k}+B u_{k}+G d_{k} \\
y_{k}^{r} & =C_{r} x_{k} \quad y_{k}=C x_{k}
\end{aligned}
$$

Step 2: Design an optimal state and disturbance estimator for $(\phi, C, G)$

This can be solved ( $M_{e}$ and $K$ can be designed) applying the modified results of [10] and [9] for LTI systems without noises:

$$
\begin{aligned}
& \bar{x}_{k}=\phi \hat{x}_{k-1}+B u_{k-1} \rightarrow \hat{x}_{k}=\bar{x}_{k}+K\left(y_{k}-C \bar{x}_{k}\right) \\
& \hat{d}_{k-1}=M_{e}\left(y_{k}-C \bar{x}_{k}\right) \rightarrow \hat{d}_{k}=A_{y_{k-1}}^{d} \hat{d}_{k-1}
\end{aligned}
$$

Here the estimation of the disturbance does not need any information about its dynamics, but if its dynamics is known $\hat{d}_{k}$ can be used in control instead of $\hat{d}_{k-1}$ and so, better results can be achieved.

An important thing is the really poor performance of the disturbance estimate in the first 5-10 time steps (it depends on the estimator dynamics). This is caused by the initially zero estimated state (see (3)). So, it is worth not to apply the first 5-10 samples of the disturbance estimate in the control! Step3: LS optimal disturbance cancellation with the control input

The task is to find a control input component which cancels most of the disturbances using their estimated value (from here ()$^{+}$stands for the left or right inverse of a rectangular matrix (depending on left or right invertibility)):

$$
\begin{aligned}
& B u_{k}^{*}=-G \hat{d}_{k} \rightarrow u_{k}^{*}=-B^{+} G \hat{d}_{k} \\
& u_{k}=\hat{u}_{k}+u_{k}^{*}=\hat{u}_{k}-B^{+} G \hat{d}_{k}
\end{aligned}
$$

The equation has an exact solution if $G=B$ otherwise this solution is only LS optimal.

Step4: Determining the solution of the zero steady state tracking error problem considering constant reference and disturbance

The equation to be solved can be constructed considering (2) and (4) (here $d_{\infty}=\hat{d}_{\infty}$ and $r_{\infty}$ denotes the constant disturbance and reference signal respectively).

$$
\begin{aligned}
& x_{\infty}=\phi x_{\infty}+B \hat{u}_{\infty}+G d_{\infty}-B B^{+} G \hat{d}_{\infty} \\
& y_{\infty}^{r}=C_{r} x_{\infty}=\underbrace{C_{r}(I-\phi)^{-1} B}_{F} \hat{u}_{\infty}+ \\
& +C_{r}(I-\phi)^{-1}\left(I-B B^{+}\right) G \hat{d}_{\infty}=r_{\infty} \\
& \hat{u}_{\infty}=F^{+} r_{\infty}- \\
& -F^{+} C_{r}(I-\phi)^{-1}\left(I-B B^{+}\right) G \hat{d}_{\infty}
\end{aligned}
$$

Here the existence of $(I-\phi)^{-1}$ is guaranteed by step 1 , and the right inverse $F^{+}$exists because $\operatorname{rank}\left(C_{r} B\right)=r$ $(r \leq m)$.

Step5/1: Derivation of the LQ optimal finite horizon solution for the centered output tracking minimax problem

The required steady state input to track a constant reference signal can be calculated using (5). However, the control of the transient from initial state to steady state should be considered. This can be designed together with the solution of cases with time varying references in a unified framework as follows.

The centered state dynamic equation results from (2), (4) and the steady state system equation (5):

$$
\begin{aligned}
& x_{k+1}=\phi x_{k}+B \hat{u}_{k}+G d_{k}-B B^{+} G \hat{d}_{k} \\
& x_{\infty}=\phi x_{\infty}+B \hat{u}_{\infty}+G d_{\infty}-B B^{+} G \hat{d}_{\infty} \\
& x_{k+1}-x_{\infty}=\phi\left(x_{k}-x_{\infty}\right)+B\left(\hat{u}_{k}-\hat{u}_{\infty}\right)+ \\
& +G\left(d_{k}-d_{\infty}\right)-B B^{+} G\left(\hat{d}_{k}-\hat{d}_{\infty}\right) \\
& \Delta \tilde{d}_{k}=G \Delta d_{k}-B B^{+} G \Delta \hat{d}_{k} \\
& \Delta x_{k+1}=\phi \Delta x_{k}+B \Delta \hat{u}_{k}+\Delta \tilde{d}_{k}
\end{aligned}
$$

The last equation in (6) gives a disturbed system dynamics around the steady state. This equation together with the centered reference signal $\Delta r_{k}=r_{k}-r_{\infty}$ can be used to form an LQ optimal minimax tracking problem for the transient (in case of constant references) or for the case with time 
varying references. The formulated problem is similar to the case in [11]. At first, the finite horizon solution should be derived considering the following functional (for the detailed functional formulation see [11])

$\Delta \tilde{x}_{k}=C_{r}^{T}\left(C_{r} C_{r}^{T}\right)^{-1} \Delta r_{k}=H \Delta r_{k}$ :

$$
\begin{aligned}
& J=\frac{1}{2} \sum_{k=0}^{N-1}\left(\left(\Delta x_{k}-\Delta \tilde{x}_{k}\right)^{T} Q\left(\Delta x_{k}-\Delta \tilde{x}_{k}\right)+\right. \\
& \left.+\Delta \hat{u}_{k}^{T} R_{u} \Delta \hat{u}_{k}-\gamma^{2} \Delta \tilde{d}_{k}^{T} R_{d} \Delta \tilde{d}_{k}\right)+ \\
& +\left(\Delta x_{N}-\Delta \tilde{x}_{N}\right)^{T} Q\left(\Delta x_{N}-\Delta \tilde{x}_{N}\right) \text { where }: \\
& Q=\bar{C}^{T} Q_{1} \bar{C}+C_{r}^{T} Q_{2} C_{r} \\
& \bar{C}=\left(I-C_{r}^{T}\left(C_{r} C_{r}^{T}\right)^{-1} C_{r}\right)
\end{aligned}
$$

Here $Q_{2}$ weights the tracking error $\left(\Delta e_{k}=\Delta y_{k}^{r}-\Delta r_{k}\right)$ and $Q_{1}$ the orthogonal projection of the state vector onto the nullspace of $C_{r}\left(\Delta \bar{y}_{k}=\bar{C} \Delta x_{k}\right)$. From this point the Lagrange multiplier method can be applied to (7) and to the last equation in (6). The costate update equation, optimal control, worst case disturbance and the structure of the costate variable results as:

$$
\begin{aligned}
& \lambda_{k}=Q\left(\Delta x_{k}-\Delta \tilde{x}_{k}\right)+\phi^{T} \lambda_{k+1} \\
& \Delta \hat{u}_{k}=-R_{u}{ }^{-1} B^{T} \lambda_{k+1} \\
& \Delta \tilde{d}_{k}^{*}=\frac{1}{\gamma^{2}} R_{d}{ }^{-1} \lambda_{k+1} \\
& \lambda_{k}=P_{k} \Delta x_{k}+S_{k} \Delta \tilde{x}_{k+1}-Q \Delta \tilde{x}_{k} \\
& \lambda_{N}=Q \Delta x_{N}-Q \Delta \tilde{x}_{N} \rightarrow P_{N}=Q, \quad S_{N}=0
\end{aligned}
$$

Finally, the Modified Riccati Difference Equation (MRDE) and an additional recursive equation results. The last expression in (9) is the expanded form of the costate variable. The optimal control and worst case disturbance can be calculated using this and (8).

$$
\begin{aligned}
& P_{k}=Q+\phi^{T} P_{k+1}[I+\underbrace{\bar{B} R^{-1} \bar{B}^{T}}_{M} P_{k+1}]^{-1} \phi \\
& S R_{k}=Q \Delta \tilde{x}_{k}+\phi^{T}\left[I+P_{k+1} \bar{B} R^{-1} \bar{B}^{T}\right]^{-1} S R_{k+1} \\
& S R_{k+1}=Q \Delta \tilde{x}_{k+1}-S_{k+1} \Delta \tilde{x}_{k+2} \\
& \bar{B}=\left[\begin{array}{ll}
B & I
\end{array}\right] \quad R=\left[\begin{array}{cc}
R_{u} & 0 \\
0 & -\gamma^{2} R_{d}
\end{array}\right] \\
& \lambda_{k+1}=P_{k+1}\left[I+M P_{k+1}\right]^{-1} \phi \Delta x_{k}- \\
& -\left[I+P_{k+1} M\right]^{-1}\left(Q H \Delta r_{k+1}-S_{k+1} H \Delta r_{k+2}\right)
\end{aligned}
$$

This completes the derivation of the minimax tracking controller for finite horizon problems. All the calculation expressions in (9) are recursive, so they need the knowledge of the reference signal on the whole horizon in advance. This difficulty should be solved considering the infinite horizon solution.

Step 5/2: Derivation of $L Q$ optimal and $L Q$ sub-optimal infinite horizon solutions

For infinite horizon the MDARE can be easily constructed from (9). Denote its solution by $P_{\infty}$. Now the generalized form of the costate variable can be written as:

$$
\begin{aligned}
& \lambda_{k+1}=P_{\infty}\left[I+M P_{\infty}\right]^{-1} \phi \Delta x_{k}- \\
& -\left[I+P_{\infty} M\right]^{-1}\left(S_{1} \Delta r_{k+1}-S_{2} \Delta r_{k+2}\right)
\end{aligned}
$$

This way $u_{k}=-R_{u}^{-1} B^{T} \lambda_{k+1}$ and $\Delta \tilde{d}_{k}^{*}=\frac{1}{\gamma^{2}} R_{d}{ }^{-1} \lambda_{k+1}$ are satisfied if one writes back $\lambda_{k+1}$ into them. To get an LQ optimal solution $S_{1}$ and $S_{2}$ should be selected to satisfy the other requirement $\lambda_{k}=Q \Delta x_{k}-Q H \Delta r_{k}+\phi^{T} \lambda_{k+1}$. Substituting the general expression for $\lambda$ (10) into this last requirement and doing some manipulations considering the last equation in (9) and assuming $\phi$ is invertible (this can be guaranteed with pole placement design in Step 1) results in the following system of equations:

$$
\begin{gathered}
P_{\infty} \Delta x_{k}=Q \Delta x_{k}+\phi^{T} P_{\infty}\left[I+M P_{\infty}\right]^{-1} \phi \Delta x_{k} \\
-S_{1} \Delta r_{k}=-Q H \Delta r_{k} \\
S_{2} \Delta r_{k+1}=-\phi^{T}\left[I+P_{\infty} M\right]^{-1} S_{1} \Delta r_{k+1} \\
0=\phi^{T}\left[I+P_{\infty} M\right]^{-1} S_{2} \Delta r_{k+2}
\end{gathered}
$$

In (11) the MDARE is written which is satisfied for all $\Delta x_{k}$. For constant reference signal (12),(13) and (14) are also satisfied and so, the obtained solution is optimal. However, unfortunately it is impossible to satisfy the last two equations for time-varying (nonzero $\Delta r_{k+2}$ ) references. So, the general LQ optimal solution of the problem is impossible. However, in real applications at time instant $k \Delta r_{k+2}$ usually should be considered with linear extrapolation because it is not known (see [11]). Considering this fact a sub-optimal selection of $S_{1}$ and $S_{2}$ is possible (defining $M_{2}=\left[I+P_{\infty} M\right]^{-1}$ ):

$$
\begin{aligned}
& \Delta r_{k+2}=2 \Delta r_{k+1}-\Delta r_{k} \\
& -S_{1} \Delta r_{k}=-Q H \Delta r_{k}-\phi^{T} M_{2} S_{2} \Delta r_{k} \\
& S_{2} \Delta r_{k+1}=-\phi^{T} M_{2} S_{1} \Delta r_{k+1}+2 \phi^{T} M_{2} S_{2} \Delta r_{k+1} \\
& \underbrace{\left[\begin{array}{cc}
I & -\phi^{T} M_{2} \\
\phi^{T} M_{2} & I-2 \phi^{T} M_{2}
\end{array}\right]}_{Z}\left[\begin{array}{c}
S_{1} \\
S_{2}
\end{array}\right]=\left[\begin{array}{c}
Q H \\
0
\end{array}\right]
\end{aligned}
$$

In (15) $Z$ is an invertible matrix. This way the sub-optimal solution for $S_{1}$ and $S_{2}$ results as:

$$
\begin{aligned}
& S_{1}=\left[I-\phi^{T} M_{2}\left(\left(I-\phi^{T} M_{2}\right)^{2}\right)^{-1} \phi^{T} M_{2}\right] Q H \\
& S_{2}=-\left(\left(I-\phi^{T} M_{2}\right)^{2}\right)^{-1} \phi^{T} M_{2} Q H
\end{aligned}
$$

Finally the control input and worst case disturbance for the centralized problem:

$$
\begin{aligned}
\Delta \hat{u}_{k} & =-K_{x 2} \Delta x_{k}+\bar{K}_{S_{1}} \Delta r_{k+1}+K_{S_{2}} \Delta r_{k} \\
\bar{K}_{S_{1}} & =K_{S_{1}}-2 K_{S_{2}} \\
K_{x 2} & =R_{u}^{-1} B^{T} P_{\infty}\left[I+\bar{B} R^{-1} \bar{B}^{T} P_{\infty}\right]^{-1} \phi \\
K_{S_{1}} & =R_{u}^{-1} B^{T}\left[I+P_{\infty} \bar{B} R^{-1} \bar{B}^{T}\right]^{-1} S_{1} \\
K_{S_{2}} & =R_{u}^{-1} B^{T}\left[I+P_{\infty} \bar{B} R^{-1} \bar{B}^{T}\right]^{-1} S_{2}
\end{aligned}
$$




$$
\begin{aligned}
\Delta \tilde{d}_{k}^{*} & =L_{x_{k}} \Delta x_{k}-\left(L_{S_{1}}-2 L_{S_{2}}\right) \Delta r_{k+1}-L_{S_{2}} \Delta r_{k} \\
L_{x_{k}} & =\frac{1}{\gamma^{2}} R_{d}^{-1} P_{\infty}\left[I+\bar{B} R^{-1} \bar{B}^{T} P_{\infty}\right]^{-1} \phi \\
L_{S_{1}} & =\frac{1}{\gamma^{2}} R_{d}^{-1}\left[I+P_{\infty} \bar{B} R^{-1} \bar{B}^{T}\right]^{-1} S_{1} \\
L_{S_{2}} & =\frac{1}{\gamma^{2}} R_{d}^{-1}\left[I+P_{\infty} \bar{B} R^{-1} \bar{B}^{T}\right]^{-1} S_{2}
\end{aligned}
$$

Step 6: The construction of the final control input signal

The final control input signal can be constructed considering (2), (4), (5), (6) and (17). The final result is:

$$
\begin{aligned}
& \tilde{u}_{k}=-K_{x} \hat{x}_{k}-K_{S_{2}}\left(r_{k+1}-r_{k}\right)+K_{r_{\infty}} r_{k+1}+ \\
& +K_{d_{\infty}} \hat{d}_{k} \text { where } K_{x}=K_{x 1}+K_{x 2} \\
& K_{r_{\infty}}=\left(K_{x 2}(I-\phi)^{-1} B+I\right) F^{+} \\
& M_{3}=\left(I-B B^{+}\right) \\
& K_{d_{\infty}}=\left[K_{x 2}(I-\phi)^{-1} M_{3}-\right. \\
& -K_{x 2}(I-\phi)^{-1} B F^{+} C_{r}(I-\phi)^{-1} M_{3}- \\
& \left.-F^{+} C_{r}(I-\phi)^{-1} M_{3}-B^{+}\right] G
\end{aligned}
$$

Note that the estimated state is used instead of the real system state, the $r_{k+1}$ reference is used with $K_{r_{\infty}}$ instead of $r_{\infty}$ and $\hat{d}_{k}$ is used instead of $\hat{d}_{\infty}$ and this provides the applicability both for constant and time-varying references and disturbances. The control input of the state and disturbance estimator $u_{k}$ should be calculated using $K_{x 2}$ instead of $K_{x}$ (and $\phi$ should be used instead of $A$ !). In the next Section the statement and proof of properties for constant references and disturbances will be done.

\section{Properties For CONSTANT REFerences AND DISTURBANCES}

Theorem 1 (Properties for const. refs. and dists.): The augmented system formulated with the proposed control method satisfies the separation principle, is asymptotically stable and guarantees zero steady state tracking error for constant reference and disturbance signals

Proof: From (3), (1) and (19) the augmented system dynamics results as follows:

$$
\begin{aligned}
& x_{k}^{e}=\hat{x}_{k}-x_{k}=(I-K C) \phi x_{k-1}^{e}=L x_{k-1}^{e} \\
& d_{k}^{e}=-A_{y_{k-1}}^{d} M_{e} C \phi x_{k-1}^{e} \\
& \underbrace{\left[\begin{array}{l}
x_{k+1} \\
x_{k+1}^{e} \\
\hat{d}_{k+1}^{e}
\end{array}\right]}_{x_{k+1}^{a}}=\underbrace{\left[\begin{array}{ccc}
\phi_{2} & -B K_{x} & B K_{d_{\infty}} \\
0 & L & 0 \\
0 & -A_{y_{k}}^{d} M_{e} C \phi & 0
\end{array}\right]}_{A^{a}}\left[\begin{array}{l}
x_{k} \\
x_{k}^{e} \\
\hat{d}_{k}
\end{array}\right]+ \\
& +\underbrace{\left[\begin{array}{c}
G \\
0 \\
A_{y_{k}}^{d}
\end{array}\right]}_{G^{a}} d_{k}+\underbrace{\left[\begin{array}{c}
-\left(B K_{S_{2}}-B K_{r_{\infty}}\right) r_{k+1}+B K_{S_{2}} r_{k} \\
0
\end{array}\right]}_{B^{a}(k)}
\end{aligned}
$$

(20) shows that the augmented system satisfies the separation principle, because neither the system state $x_{k}$ nor the reference terms $r_{k+1}, r_{k}$ affect the dynamics of the state and disturbance estimator. The stability of the augmented state matrix $A^{a}$ can be easily proven. Calculate now the steady state of the system with constant references and disturbances (of course $r_{k+1}=r_{k}=r_{\infty}=$ const, $d_{k+1}=d_{k}=d_{\infty}=$ const and $A_{y_{k}}^{d}=I$ should be substituted).

$$
\begin{aligned}
& x_{\infty}^{a}=\underbrace{\left(A^{a}\right)^{\infty} x_{0}^{a}}_{=0}+ \\
& +\left(I-A^{a}\right)^{-1} G^{a} d_{\infty}+\left(I-A^{a}\right)^{-1} B^{a}(\infty)
\end{aligned}
$$

(21) shows that the system is asymptotically stable for finite, constant references and disturbances. From (1), (19), (20) and (21) a straightforward calculation leads to:

$$
\left[\begin{array}{c}
y_{\infty}^{r}=C_{r} x_{\infty} \\
x_{\infty}^{e} \\
\hat{d}_{\infty}
\end{array}\right]=\left[\begin{array}{c}
r_{\infty} \\
0 \\
d_{\infty}
\end{array}\right]
$$

This shows that the steady state estimation error is zero, the estimated disturbance equals the real disturbance in steady state and the zero tracking error is guaranteed.

Theorem 2 (Finite functional value): The functional with constant reference and disturbance values is finite even considering infinite horizon.

Proof:

The proof is straightforward from the definition of the terms in the functional:

$$
\begin{aligned}
& J(\Delta x, \Delta \tilde{x}, \Delta \hat{u}, \Delta \tilde{d})= \\
& =\frac{1}{2} \sum_{k=0}^{\infty}\left(\left(\Delta x_{k}-\Delta \tilde{x}_{k}\right)^{T} Q\left(\Delta x_{k}-\Delta \tilde{x}_{k}\right)+\right. \\
& \left.+\Delta \hat{u}_{k}^{T} R_{u} \Delta \hat{u}_{k}-\gamma^{2} \Delta \tilde{d}_{k}^{T} R_{d} \Delta \tilde{d}_{k}\right) \\
& \text { here: } \\
& \Delta x_{k}=x_{k}-x_{\infty} \rightarrow 0 \text { as } k \rightarrow \infty \\
& \Delta \hat{u}_{k}=\hat{u}_{k}-\hat{u}_{\infty} \rightarrow 0 \text { as } k \rightarrow \infty \\
& \Delta \tilde{x}_{k}=H \Delta r_{k}=H\left(r_{k}-r_{\infty}\right)=0 \forall k \\
& \Delta \tilde{d}_{k}=\underbrace{G\left(d_{k}-d_{\infty}\right)}_{=0 \forall k}-B B^{+} G\left(\hat{d}_{k}-\hat{d}_{\infty}\right) \\
& \rightarrow 0 \text { as } k \rightarrow \infty
\end{aligned}
$$

This way the functional in (23) describes the well known minimax regulator problem, where the states and worst case disturbances go to zero together with the control input and this way the value of the infinite horizon functional is finite.

\section{THE SIMULATION EXAMPLES}

The following simple, discrete time (and its continuous time equivalent) longitudinal quadrotor dynamical model was considered in simulations (linearized model around hovering): 


$$
\begin{aligned}
& A=\left[\begin{array}{cccc}
0.9994 & 0 & -1.95 e-5 & -0.0196 \\
0 & 0.9992 & 0 & 0 \\
0 & 0 & 0.9815 & 0 \\
0 & 0 & 0.0020 & 1.0000
\end{array}\right] \\
& B=\left[\begin{array}{cc}
-8.79 e-7 & 8.79 e-7 \\
-1.71 e-3 & -1.71 e-3 \\
0.134 & -0.134 \\
1.34 e-4 & -1.34 e-4
\end{array}\right] \\
& G=\left[\begin{array}{cc}
-1.54 e-3 & 0 \\
0 & -2.23 e-3 \\
0 & 0 \\
0 & 0
\end{array}\right] \\
& C=\left[\begin{array}{llll}
1 & 0 & 0 & 0 \\
0 & 1 & 0 & 0 \\
0 & 0 & 1 & 0
\end{array}\right] \\
& x^{T}=\left[\begin{array}{llll}
u & w & Q & \theta
\end{array}\right] \quad d^{T}=\left[\begin{array}{ll}
d_{u} & d_{w}
\end{array}\right]
\end{aligned}
$$

The sample time was $T=0.002 \mathrm{sec}$ which results from the model simplicity (neglecting motor dynamics for example). The bandwidth of more complex guadrotor models is smaller. The elements of the state vector are: $u, w$ velocity components and $Q$ angular rate in body coordinate system (coord. sys.), and $\theta$ pitch angle in earth coord. sys. The disturbance vector considers wind disturbance on $u$ and $w$. Its transformation from wind (earth) coord. sys. to body is neglected because of small $\theta$ angles. The control goal is to track a given $u_{r e f}$ velocity and $Q_{r e f}=0$ angular rate with rejecting the wind disturbances which means forward flight.

Two tracking controllers were designed for this model. The first uses the method proposed here (further denoted by $\operatorname{MM}(=$ Minimax $)$ method), the second is an $\mathcal{H}_{\infty}$ tracker designed for disturbance rejection.

The latter could be designed only by completing the system with the integral of $e_{u}(k)=u(k)-u_{r e f}(k)$ tracking error as a state and considering $Q_{r e f}=0$ reference which means that it is enough to stabilize $Q$, tracking error minimization is not required. Otherwise, completing also with the integral of $e_{Q}(k)=Q(k)-Q_{r e f}(k)$ the resulting augmented system was not controllable with the input and so, the design task was unfeasible. An LMI based continuous time solution was obtained and the resulting controller was discretized after.

The other controller was designed following the proposed 6 steps (see Section II). During the design the MDARE should be solved with $\gamma$ iteration using the so called bisection algorithm as in the continuous time (CT) case. But the MDARE should be solved using the augmented input matrix $\bar{B}$ (see (9)) and this way it considers also the worst case disturbance as a useful input applicable to stabilize the system. This can result in an unstable system at the achieved minimum $\gamma$ value if one does not generate also the worst case disturbance as a control input. But in real applications the generation of worst case disturbance as an input is usually impossible (such as here). This problem is pointed out also in [12] for CT minimax control. The solution similar to the one proposed in [12] is to do $\gamma$ iteration not for the solvability or unsolvability of the MDARE but for the stability or instability of $\phi-B K_{x 2}$ instead. This way larger final gamma value results, but the controlled system will be stable purely with the control input (the worst case disturbance is not needed).

The achieved $\gamma$ value in $\mathcal{H}_{\infty}$ design is 0.812 which is good for disturbance rejection and tracking. With MM method $\gamma=$ 592.3178 resulted which is a huge value but small enough to reject the small disturbance residuals as can be seen from simulation results.

Both two methods were tested with $u_{r e f}=1$ (using a ramp transient from 0 to 1) $Q_{r e f}=0, d_{u}=0.5625$ and $d_{w}=0.04$. This latter two are converted values related to $-0.25 \mathrm{~m} / \mathrm{s}$ wind along $\mathrm{X}$, and $0.2 \mathrm{~m} / \mathrm{s}$ along $\mathrm{Z}$ axis of earth coord. sys. (the conversion is needed because a simplified air drag calculation formula was used to determine the elements of $G)$.
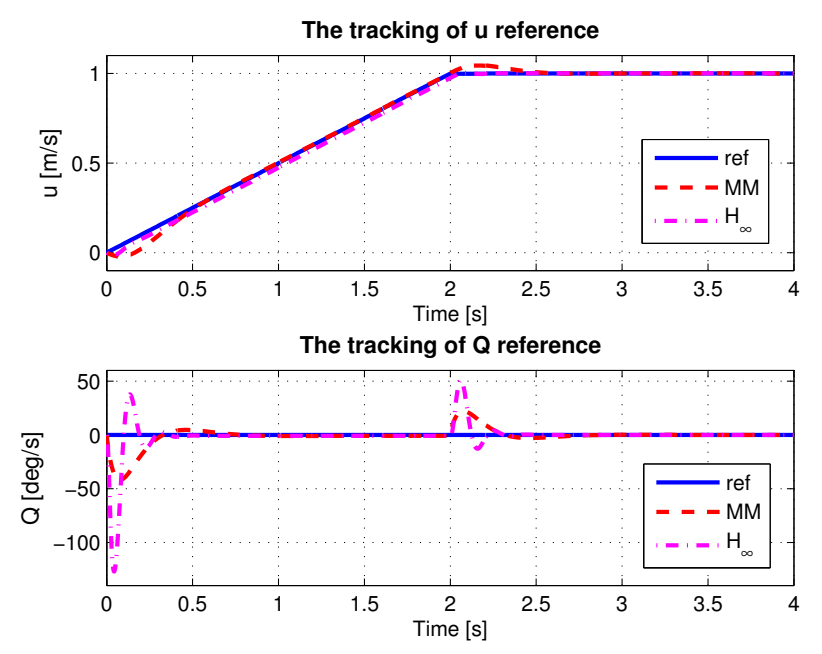

Fig. 1. The tracking of references
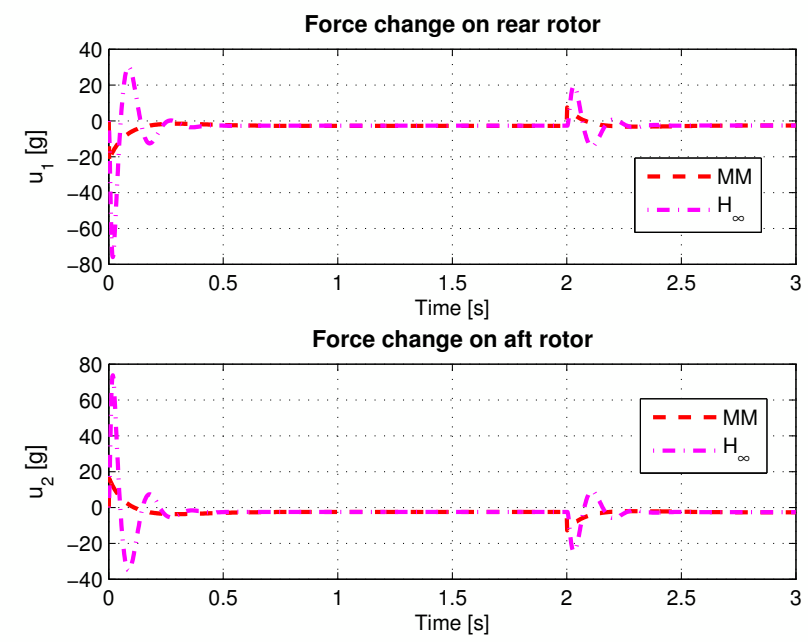

Fig. 2. Control inputs

The simulation results can be seen in Figures 1 and 2 . Both methods were capable to well track the $u$ reference 
and set $Q$ to zero rejecting the disturbances (see Figure 1). In the application of MM method the control input component generated by $K_{S_{2}}$ (see (19)) was originally very large and needed saturation. With the proper selection of saturating values the tracking could be tuned to achieve acceptable control inputs and good tracking performance. This property gives an extra degrees of freedom in the application of the design.

The two methods can be compared considering the tracking performance and required control input. The tracking performances can be seen in Figure 1 . The $\mathcal{H}_{\infty}$ controller tracks $u$ with a small almost constant error, meanwhile the MM method has larger tracking errors where the slope of $u_{\text {ref }}$ changes, but almost zero error at the linear sections. The truncated two norms of the errors are the following (considering 10sec simulation):

$E_{u}=\sqrt{\sum_{k=0}^{N} e_{u}(k)^{2}}, \quad E_{Q}=\sqrt{\sum_{k=0}^{N} e_{Q}(k)^{2}}$
$E_{u}^{\mathcal{H} \infty}=0.8078, E_{u}^{M M}=1.0117$

The norm is a bit larger in case of MM method, but consider also the results for $Q$ :

$E_{Q}^{\mathcal{H}_{\infty}}=11.7983, E_{Q}^{M M}=6.7346$

this case both the norm and the extremal values are better for

MM method (see Figure 1). MM generates smaller $Q$ rates which is good for the quadrotor structure and can make it possible to stay in the linear range around hovering state. The steady state $e_{u}$ and $e_{Q}$ values are also better with MM method: after $11 \mathrm{sec}$ :

$e_{u}^{M M}=1.55 e-14, e_{Q}^{M M}=2.7 e-15$

meanwhile with $\mathcal{H}_{\infty}$ method after $20 \mathrm{sec}$ :

$e_{u}^{\mathcal{H}_{\infty}}=1 e-5, e_{Q}^{\mathcal{H}_{\infty}}=2 e-8$

The comparison of the control inputs also gives better results with MM. The maximum control input signals are smaller for MM (see Figure 2) and also the truncated two norms are smaller (for $10 \mathrm{sec}$ ):

$E_{u_{\infty}}^{\mathcal{H}}=3.5148, E_{u_{1}}^{M M}=2.052$

$E_{u_{2}}^{\mathcal{H}}=3.5455, E_{u_{2}}^{M M}=1.985$

As a conclusion it can be said that the tracking performance is almost the same for the two methods (considering only $\mathrm{u}$ ), but the used amount of control energy and the extremal values of $Q$ are better for MM method. Another advantage of MM method is the need to represent only a 4 state dynamical controller (state and disturbance estimator see [10].) instead of an 8 state resulting from the augmented plant of the $\mathcal{H}_{\infty}$ design.

\section{CONCLUSIONS}

This paper presents an LQ optimal minimax tracking solution for DT, LTI systems with deterministic disturbances. The solution can be designed with a multi step method containing stabilization with state feedback, state and disturbance estimation, LS optimal disturbance cancellation, steady state tracking solution, centering of original system dynamics and minimax tracker design. The final required control input is the sum of components designed during the different steps. The properties for constant references and disturbances are stated and proven (satisfaction of the separation principle, asymptotic stability, zero steady state tracking error and finite functional value on infinite horizon).

The method was compared with an $\mathcal{H}_{\infty}$ optimal tracker applied to the control of a quadrotor longitudinal model. The goal was to change from hovering to forward flight. The velocity tracking properties of the two methods are almost the same, but the proposed new method used less control energy, induced smaller angular rates and requires to implement only a 4 state estimator instead of the 8 state $\mathcal{H}_{\infty}$ controller.

Both methods were tested also for time varying $u$ reference giving promising results. However, the examination of the properties of proposed method with time-varying references and disturbances will be the topic of another article.

The introduction of state and measurement noises can highly affect these noiseless results so, this should be later also examined, together with the robustness properties and possible extension to time-varying systems.

\section{REFERENCES}

[1] J. L. Willems and I. M. Y. Mareels, A Rigorous Solution of the Infinite Time Interval LQ Problem with Constant State Tracking, Sys. \& Cont. Let., vol. 52, 2004, pp 289-296.

[2] C. Choi and T-C. Tsao, $\mathcal{H}_{\infty}$ Preview Control for Discrete-Time Systems, J. of Dyn. Sys. Meas. and Contr., vol. 123, 2001, pp. 117-124.

[3] G. Tadmor and L. Mirkin, " $\mathcal{H}_{\infty}$ Preview Control and Fixed-Lag Smoothing II: Fixed Size ARE Solutions in Discrete-Time Systems", in Proc. of 42nd IEEE Conference on Decision and Control, Maui, Hawai, USA, 2003, pp. 6521-6526.

[4] G. Tadmor and L. Mirkin, $\mathcal{H}_{\infty}$ Control and Estimation with PreviewPart II: Fixed Size ARE Solutions in Discrete-Time, IEEE Trans. on Aut. Contr., vol. 50, No. 1, January 2005, pp. 29-40.

[5] K. Takaba, "A Tutorial on Preview Control Systems", in Proc. of SICE Annual Conference in Fukui,Fukui University, Japan, pp. 1388-1393.

[6] A. Kojima, "Generalized preview and delayed $\mathcal{H}_{\infty}$ control: output feedback case", in Proc. of 44th IEEE Conference on Decision and Control, and the European Control Conference 2005, Seville, Spain, 2005, pp. 5770-5775.

[7] K. Zhou, J. C. Doyle and K. Glover, Robust and Optimal Control, Prentice-Hall, Inc.; 1996.

[8] B-S. Chen, C-S. Whu and H-J. Uang, A Minimax Tracking Design for Wheeled Vehicles with Trailer Based on Adaptive Fuzzy Elimination Scheme, IEEE Trans. on Contr. Sys. Techn., vol. 8, No. 3, May 2000, pp. 418-434.

[9] M. Darouach and M. Zasadzinski, Unbiased Minimum Variance Estimation for Systems with Unknown Exogenous Inputs, Automatica, vol. 33, No. 4, 1997, pp. 717-719.

[10] S. Gillijns and B. De Moor, Unbiased minimum-variance input and state estimation for linear discrete-time systems, Automatica, vol. 43, 2007, pp. 111-116.

[11] P. Bauer, B. Kulcsar and J. Bokor, "An Exact Solution for the Infinite Horizon LQ Optimal Output Tracking Problem", in Proc. of 17th IEEE International Conference on Control Applications Part of 2008 IEEE Multi-Conference on Systems and Control, San Antonio, Texas, USA, 2008, pp. 822-827.

[12] L. Kovacs and B. Palancz, Glucose-Insulin Control of Type1 Diabetic Patients in $\mathcal{H}_{2} / \mathcal{H}_{\infty}$ Space Via Computer Algebra, AB2007, Springer Lecture Notes in Computer Science, vol. 4545, 2007, pp. 95-109. 